\title{
General practice deprivation payments: are rural practices disadvantaged?
}

\author{
D O’Reilly, K Steele
}

The use of the Jarman under privileged area (UPA) scoring system as a means of remunerating general practitioners (GPs) for the additional workload associated with deprivation has been the subject of much debate and criticism. ${ }^{1}$ GPs who are based in rural practices often fail to attract any deprivation payments and are particularly aggrieved. They criticise the UPA system for being more sensitive to urban deprivation and therefore inherently biased against rural practice. ${ }^{2}$

Electoral wards (EWs) are currently the geographical unit at which Jarman scores are calculated and deprivation payments allocated. However, in the original paper ${ }^{3}$ Jarman argued that enumeration districts (EDs) were more appropriate, a view supported more recently by others. ${ }^{4}$ This paper asks if the current system fails to detect disadvantage, particularly in rural areas, because it is based at EW rather than ED level.

\section{Methods and results}

A Jarman score was calculated for each of the 566 EWs and 3729 EDs in Northern Ireland (because of a more complete census coding this is possible in the province, unlike most other parts of the UK). These were then assigned to high, medium, low or no payment bands. The ED based system identified a wider range of deprivation scores and so to maintain the same proportion of the population and deprivation payments allocated to each band, different Jarman score cut offs had to be used to define the bands. These were high $(>55.70)$, middle ( $>$ 42.92), low (>32.50).

The size of each EW at the time of the 1991 census was used to determine its density in terms of persons per hectare. These were divided into four categories (very sparse $(<0.80)$, sparse $(<7.84)$, dense $(>7.84)$, and very dense $(>27.4)$ ) such that each contained a quarter of the Northern Ireland population. EDs were allocated to the density category of their parent electoral ward irrespective of their

Table Numbers of population in sparsely and densely populated areas attracting deprivation payments according to whether the allocation to whether the allocation is made at ED or EW level

\begin{tabular}{|c|c|c|c|c|c|c|c|c|}
\hline \multirow[b]{4}{*}{ Payment band } & \multicolumn{8}{|c|}{ Density of area (persons per hectare) } \\
\hline & \multicolumn{2}{|c|}{ Very sparse } & \multicolumn{2}{|l|}{ Sparse } & \multicolumn{2}{|l|}{ Dense } & \multicolumn{2}{|l|}{ Very dense } \\
\hline & \multicolumn{2}{|l|}{ Allocation } & \multicolumn{2}{|l|}{ Allocation } & \multicolumn{2}{|l|}{ Allocation } & \multicolumn{2}{|l|}{ Allocation } \\
\hline & at $E D$ & at $E W$ & at $E D$ & at $E W$ & at $E D$ & at $E W$ & at $E D$ & at $E W$ \\
\hline High & 448 & 0 & 8834 & 6295 & 10887 & 3983 & 23964 & 34352 \\
\hline Medium & 1709 & 0 & 18399 & 10201 & 18960 & 24147 & 35544 & 40157 \\
\hline Low & 13015 & 0 & 22030 & 16904 & 28256 & 40084 & 40230 & 46561 \\
\hline Any payment & 15172 & 0 & 49263 & 33400 & 58103 & 68214 & 99738 & 121070 \\
\hline No payment & 379982 & 395154 & 346855 & 362718 & 334246 & 324135 & 294477 & 273145 \\
\hline Funding attracted & $£ 99532$ & $£ 0$ & $£ 380679$ & $£ 254594$ & $£ 446040$ & $£ 485721$ & $£ 795290$ & $£ 981226$ \\
\hline
\end{tabular}

own density. The numbers and proportions of the population that attract general practitioner deprivation payments within each of the four density categories were calculated and the difference in distribution determined according to whether the allocation was made at EW or at ED level.

Under an EW based allocation system no patient living in the most sparsely populated one quarter of wards in Northern Ireland attracts a deprivation payment (table 1). Alternatively $30.7 \%$ of the population in the most densely populated EWs attract some payments and approximately one in 12 of these patients attract payment at the "high" band.

If EDs rather than EWs are used as the basis of allocation of GP deprivation payments, different parts of the population become eligible to attract payments and a redistribution of funds occurs. The association between payments and population density is still very evident though the gradient is attenuated. The most densely populated one quarter of wards would lose approximately $£ 186000(18.9 \%)$ and "sparse" areas would attract nearly $50 \%$ as much funds as they presently do.

\section{Comment}

There are many possible ways to define rurality. ${ }^{2}$ This paper uses the arguably cruder but more readily understandable concept of population. The data presented would tend to support the hypothesis that there are areas within rural areas that are at least as deprived as some of those in more densely populated areas. These areas are not recognised under the current EW based allocation system but would be under an ED based system. Basing deprivation payments on EDs rather than EWs would therefore provide a more equitable distribution and reduce the present rural disadvantage. Calculating Jarman scores at ED level may pose problems in England and Wales but not in Northern Ireland where $100 \%$ of census forms have been coded. ${ }^{5}$
Care Research Unit, Belfast

D O'Reilly

Department of

General Practice, Queens University of Belfast

K Steele

Correspondence to: Dr O'Reilly, Health and

Health Care Research Unit, Mulhouse Building, Grosvenor Road, Belfast BT12 6BJ.

Accepted for publication 9 February 1998 
Funding: none.

Conflicts of interest: none.

1 Carr Hill RA, Sheldon T. Designing a deprivation payment for general practitioners: the UPA (8) wonderland. $B M Y$ $393-6$

2 Royal College of General Practitioners. Rural General Practice in the United Kingdom. Occasional Paper 71. London: RCGP, 1995.
3 Jarman B. Identification of underprivileged areas. BMF 1983;312:1705-9.

4 Hastings A. Deprivation payments should be based on enumeration districts. BMF 1996;312:183-4.

5 Jarman B. Scores should be based on enumeration districts and payments should be phased in gradually. BMF 1997;314:228-9.

$c$

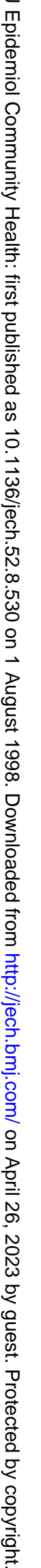

